\title{
BMJ Open Rapid paediatric fluid resuscitation: a randomised controlled trial comparing the efficiency of two provider-endorsed manual paediatric fluid resuscitation techniques in a simulated setting
}

\author{
Evan T Cole, ${ }^{1}$ Greg Harvey, ${ }^{1}$ Sara Urbanski, ${ }^{1}$ Gary Foster, ${ }^{2,3}$ \\ Lehana Thabane, ${ }^{1,2,3,4}$ Melissa J Parker ${ }^{1,5}$
}

To cite: Cole ET, Harvey G, Urbanski S, et al. Rapid paediatric fluid resuscitation: a randomised controlled trial comparing the efficiency of two provider-endorsed manual paediatric fluid resuscitation techniques in a simulated setting. BMJ Open 2014:4:e005028.

doi:10.1136/bmjopen-2014005028

- Prepublication history for this paper is available. To view please visit the journal (http://dx.doi.org/10.1136/ bmjopen-2014-005028).

Received 10 February 2014 Revised 13 June 2014 Accepted 17 June 2014

CrossMark

For numbered affiliations see end of article.

Correspondence to Dr Melissa J Parker; parkermj@mcmaster.ca

\section{ABSTRACT}

Objectives: Manual techniques of intravascular fluid administration are commonly used during paediatric resuscitation, although it is unclear which technique is most efficient in the hands of typical healthcare providers. We compared the rate of fluid administration achieved with the disconnect-reconnect and push-pull manual syringe techniques for paediatric fluid resuscitation in a simulated setting.

Methods: This study utilised a randomised crossover trial design and enrolled 16 consenting healthcare provider participants from a Canadian paediatric tertiary care centre. The study was conducted in a non-clinical setting using a model simulating a $15 \mathrm{~kg}$ child in decompensated shock. Participants administered $900 \mathrm{~mL}(60 \mathrm{~mL} / \mathrm{kg})$ of normal saline to the simulated patient using each of the two techniques under study. The primary outcome was the rate of fluid administration, as determined by two blinded independent video reviewers. We also collected participant demographic data and evaluated other secondary outcomes including total volume administered, number of catheter dislodgements, number of technical errors, and subjective and objective measures of provider fatigue.

Results: All 16 participants completed the trial. The mean (SD) rate of fluid administration $(\mathrm{mL} / \mathrm{s})$ was greater for the disconnect-reconnect technique at 1.77 $(0.145)$ than it was for the push-pull technique at 1.62 (0.226), with a mean difference of $0.15(95 \% \mathrm{Cl} 0.055$ to $0.251 ; p=0.005)$. There was no difference in mean volume administered $(p=0.778)$ or participant selfreported fatigue $(p=0.736)$ between techniques. No catheter dislodgement events occurred.

Conclusions: The disconnect-reconnect technique allowed for the fastest rate of fluid administration, suggesting that use of this technique may be preferable in situations requiring rapid resuscitation. These findings may help to inform future iterations of paediatric resuscitation guidelines.

Trial registration number: This trial was registered at ClinicalTrials.gov [NCT01774214] prior to enrolling the first participant.

\section{Strengths and limitations of this study}

- A randomised crossover trial design was the most rigorous method to address the research question.

- Findings provide objective data for clinicians who must select a method by which to perform rapid paediatric fluid administration, and may help inform future resuscitation guidelines.

- Limitations of this research include use of a nonclinical model as a patient surrogate and that we did not evaluate set-up time for each technique.

- The clinical significance of our findings is unknown.

\section{INTRODUCTION}

\section{Background}

Paediatric shock is a recognised medical emergency which, when left untreated, inevitably leads to further deterioration and cardiac arrest. ${ }^{1}$ For children in particular, hypotension is a late clinical finding, which requires immediate action to avert disaster. ${ }^{2}$ Current resuscitation guidelines recommend rapid intravascular administration of isotonic crystalloid and/or colloid as an essential component of the initial resuscitation and stabilisation of paediatric shock. ${ }^{3-6}$

The American College of Critical Care Medicine (ACCM) guidelines for haemodynamic support in paediatric septic shock recommend that fluid boluses of $20 \mathrm{~mL} / \mathrm{kg}$ be initiated immediately and repeated until perfusion is restored or signs of fluid overload develop. ${ }^{7}$ Although the FEAST trial has led to questions regarding the role of fluid resuscitation in paediatric shock management, it is far from clear that these findings should be extrapolated to the European and North American clinical settings where anaemia and malaria are comparatively 
rare $^{8}{ }^{9}$ Guidelines therefore continue to recommend prompt and rapid fluid administration for the treatment of paediatric shock in these settings, ${ }^{10}$ as this has been linked with improved survival odds. ${ }^{11-15}$

\section{Importance}

Although various paediatric resuscitation guidelines recommend rapid intravascular fluid administration, there is a paucity of evidence regarding how to best achieve this in the clinical setting. Recommended benchmarks for timely fluid administration are often not met in practice, suggesting that further research to improve knowledge translation is warranted. ${ }^{12} 131617$ Survey data from one Canadian paediatric academic centre demonstrate that acute care providers use a number of techniques to perform fluid resuscitation for children, with manual syringe techniques being most common. ${ }^{18}$

There are two commonly used syringe techniques for manual fluid resuscitation: the disconnect-reconnect technique (DRT, figure 1) and the push-pull technique (PPT, figure 2). Although previous research has separately evaluated these two techniques, ${ }^{19}{ }^{20}$ their relative efficiency in the hands of typical healthcare providers has not been studied.

\section{Goals of this investigation}

The primary objective of this study was to compare two commonly used manual fluid resuscitation techniques (DRT and PPT) to determine which facilitates a faster rate of fluid administration in a simulated paediatric resuscitation scenario. We also evaluated additional outcomes relevant to overall fluid resuscitation efficiency.

\section{METHODS}

Full trial protocol was published prior to start of trial. ${ }^{21}$ The following represents an abbreviated version of the trial protocol.

\section{Study design and setting}

The study was a single-blind, non-clinical, randomised crossover trial with two study arms. The trial was conducted at McMaster Children's Hospital, an academic tertiary paediatric care centre in Hamilton, Canada. Approval for study conduct was obtained from the Hamilton Integrated Research Ethics Board, and trial registration with ClinicalTrials.gov (NCT01774214) was completed prior to enrolment of the first study participant. All participants provided written informed consent and participated voluntarily.

\section{Selection of participants}

Eligible participants included nursing staff, physicians and medical trainees at McMaster Children's Hospital who would be expected to perform manual fluid resuscitation as part of their clinical activities. Participants were
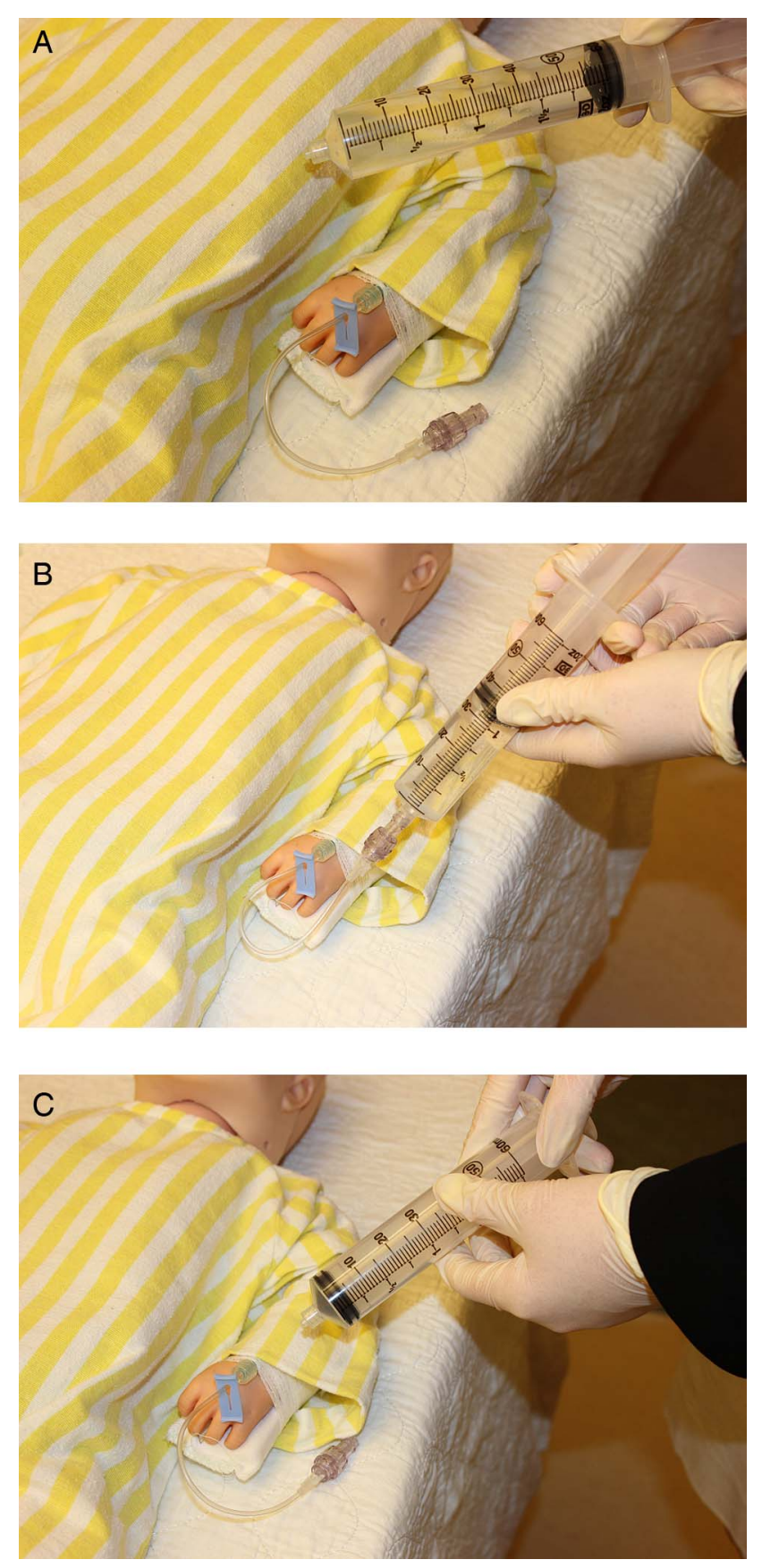

Figure 1 (A-C) The 'Disconnect-reconnect' technique (DRT) for fluid administration requires an assistant. The assistant prepares syringes of fluid while the provider repeatedly selects a syringe $(A)$, attaches it to the intravenous line and depresses the plunger $(B)$, then disconnects and discards the empty syringe $(C)$.

to be excluded if they had poor English, physical limitations affecting performance of the required tasks or had acted in a physically strenuous capacity (eg,providing CPR) in the $30 \mathrm{~min}$ preceding participation. Coffee gift cards ( $\$ 25$ value) were offered to all participants as a participation incentive, with a second coffee card available as a prize to the participant in each group with the best performance. 

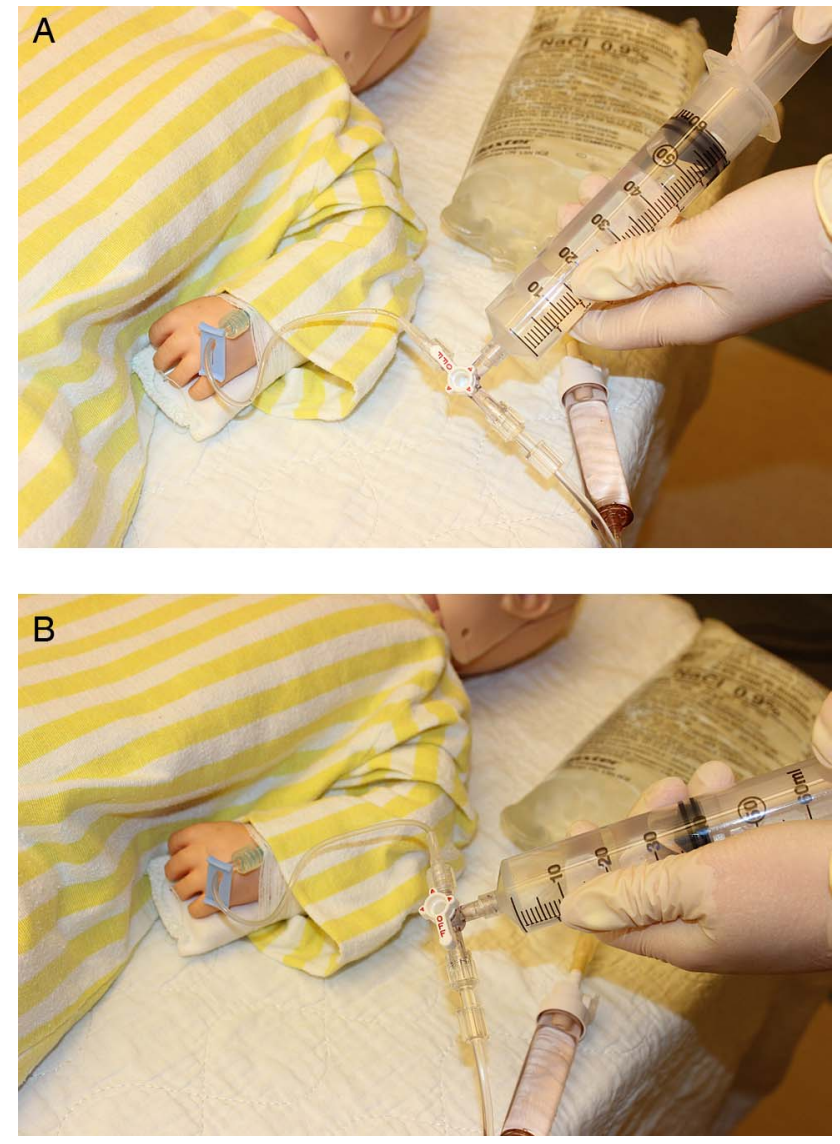

Figure 2 (A and B) The 'push-pull' technique (PPT) for fluid administration requires the healthcare provider to repeatedly perform two steps. With the stopcock positioned 'off' to the patient, the provider first pulls on the syringe plunger to fill the syringe with fluid (A). The provider must then toggle the stopcock 'on' to the patient and depress the plunger to administer fluid to the patient $(B)$.

\section{Model setup}

The trial setup included a model simulating a $15 \mathrm{~kg}$ child with a 1 in 22-gauge, intravenous catheter. The distal end of the catheter was secured in an unobstructed manner within conduit tubing leading to a graduated cylinder. The graduated cylinder, used to collect and measure fluid effectively delivered to the model, was kept hidden from the view of participants. The proximal end of the intravenous catheter was connected via a catheter extension set to the appropriate technique-dependent setup. For the DRT method an excess supply of empty $60 \mathrm{~mL}$ syringes was provided, as well as a $1 \mathrm{~L}$ bag of normal saline attached appropriately to a needle-less syringe adaptor. For the PPT setup, the proximal end of the catheter extension set was connected to a triple stopcock, with an empty $60 \mathrm{~mL}$ syringe at the second port, and standard intravenous tubing leading to a $1 \mathrm{~L}$ bag of normal saline at the third port.

\section{Randomisation}

A third party randomisation technique was used to assign participants to one of the two study arms in a 1:1 ratio, determining the order in which the two interventions were performed. The allocation sequence was generated by the third party, who was also responsible for the randomisation of participants, and for keeping the randomisation schedule secret from and inaccessible to the study investigators. Simple randomisation was utilised, with no blocking or stratification.

\section{Blinding}

We undertook a number of measures to effect blinding and minimise the risk of bias. Participants were provided with trial details sufficient to allow for informed consent, but they were not advised of the study outcomes of interest or the hypotheses of the investigators. Participants were blinded to the amount of fluid accumulating in the graduated cylinder by physically shielding it during testing. The research assistant was provided limited detail regarding the purpose of the study as necessary to facilitate recruitment and consent of participants, set-up of required equipment and coordination of testing. While the randomisation schedule was concealed, the research assistant could not be blinded to participant assignment due to the nature of the intervention. The beginning and end of the intervention phase was defined by start and stop signals. The verbal start signal was an instruction given by the research assistant to the participant, while the verbal stop signal was given by the participant when they felt they had administered the required amount of fluid. It is conceivable that the research assistant was aware of the purpose of the trial, although there were no conflicts of interest with respect to study outcomes.

To minimise risk of bias in ascertainment of the primary outcome, we also utilised two independent outcome assessors who were blinded to the purpose of the study. The outcome assessors, who were nonclinicians, independently extracted the intervention time outcome data based on review of video footage displaying only the graduated cylinder and in which the start and stop announcements were audible. The outcome assessors also reviewed video footage of each intervention filmed from a second angle, extracting information on technical errors. As the videos were numbered by participant ID, and the outcome assessors reviewed all video footage, it is possible that they could have deduced the interventions under study and participant assignment. Outcome data were extracted independently and in duplicate to increase surety. With these measures, we considered our trial as single blind. Collected data were input by EC.

\section{Intervention and participant testing}

The study intervention involved administration of $60 \mathrm{~mL} / \mathrm{kg}(900 \mathrm{~mL})$ of normal saline to the simulated patient as rapidly as possible using DRT or PPT. Pursuant to the crossover design, each of the two interventions was applied in turn to each participant, with a 
washout time of $30 \mathrm{~min}$ to mitigate any potential impact of fatigue on subsequent performance.

After obtaining informed consent, participants were randomised and underwent a standardisation procedure to orient them to the study procedures and techniques to be performed. ${ }^{21}$ The participant was then provided with a clinical vignette for the simulated scenario describing a hypotensive child with suspected septic shock. Formal evaluation was initiated on verbal prompt from the research assistant. Participants were required to recall the volume of fluid that they had administered and completed the intervention when they believed $900 \mathrm{~mL}$ had been given.

\section{Data collection and outcomes}

All testing was directly observed by the research assistant and video recorded. Two video cameras captured video data during testing: Camera 1 filmed the catheter site where fluid was being administered by the participant, while Camera 2 filmed the graduated cylinder where fluid accumulated over the course of the intervention. Two outcome assessors who were not informed of the purpose of the trial independently reviewed the videos for specific outcome data (below). The primary outcome was fluid administration rate (total fluid administered/total time). Total intervention time was determined by the video assessors, based on the 'start' and 'stop' signals from the research assistant and participant, respectively. The research assistant determined the total volume administered on completion of the intervention.

\section{Secondary outcomes included}

1. Total volume effectively administered as a measure of technique accuracy.

2. Interval rates of fluid administration for the first, second and third $20 \mathrm{~mL} / \mathrm{kg}$ aliquots administered.

3. Self-reported fatigue as determined from a seven-point Likert scale on a postintervention questionnaire.

4. The proportion of catheter dislodgement events that occurred.

5. Technical issues encountered during the intervention that resulted in a significant departure from intended procedural technique.

\section{Sample size}

For the purposes of sample size calculation, infusion time and SD data from our previously completed DRT trial $^{19}$ were used as nuisance parameters. The POWER procedure in SAS(r) V.9.2 statistical software was used to calculate a required sample size of 16 to detect a mean difference of $0.2 \mathrm{~mL} / \mathrm{s}$ (deemed significant based on clinical experience of investigators), with power 0.9 and $\alpha=0.05$.

\section{Analysis}

The reporting of the trial was carried out in accordance with the CONSORT criteria (http://www.consort-statement. org). We used a flow diagram to summarise the flow of participants in the study. The baseline characteristics are analysed using descriptive statistics reported as count (per cent) for each categorical variable. We planned to perform all analyses according to an intention-to-treat basis. The primary outcome was analysed by a two-tailed paired Student $t$ test. Differences in volume of fluid effectively administered were evaluated with a two-tailed paired Student $t$ test. Interval rates of fluid administration were analysed with a repeated measures analysis of variance. Self-reported fatigue comparisons were analysed using a Wilcoxon signed-rank test. We planned to compare the proportion of catheter dislodgement events using McNemar's test. The criterion for statistical significance was set at $\alpha=0.05$. The results are reported as estimate of effect, 95\% CI and associated p value. All analyses were performed using IBM SPSS Statistics V.20 (Illinois, Chicago, USA).

\section{RESULTS}

\section{Characteristics of study participants}

Sixteen eligible healthcare providers (table 1) were consented for testing with no excluded participants. All participants completed the assigned interventions and questionnaire as per protocol, and were included in the final analysis (figure 3). Enrolment and testing were completed between April and June 2013.

\section{Main results}

Outcome analysis results are summarised in table 2. The primary outcome of total fluid administration rate $(\mathrm{mL} / \mathrm{s})$ significantly differed between the two techniques, with a mean difference of 0.15 (95\% CI 0.05 to $0.25 ; \mathrm{p}=0.005$ ). DRT was more efficient with a mean (SD) fluid administration rate $(\mathrm{mL} / \mathrm{s})$ of $1.77(0.145)$ compared with 1.62 (0.226) for PPT. Of note, one participant's administration rate was a significant outlier in the PPT group. Exclusion of this outlier from analysis did not significantly impact the primary outcome result.

There was no difference in the volume $(\mathrm{mL})$ of fluid effectively administered, -6.25 (95\% CI -52.76 to 40.26 ; $\mathrm{p}=0.778$ ), with mean volumes for each group close to the $900 \mathrm{~mL}$ target (DRT (891, SD 36.6) and PPT (898, SD 58.1)).

A significant change in fluid administration rate occurred over the DRT intervention $(p<0.001)$ and the PPT intervention $(p=0.003)$. Pairwise comparisons of mean $(\mathrm{SD})$ infusion rates $(\mathrm{mL} / \mathrm{s})$ were performed. DRT Rate 1, 1.63 (0.143) was significantly different from Rate 2, 1.83 (0.176) and Rate 3, 1.88 (0.180); $(\mathrm{p}<0.001)$, while Rates 2 and 3 did not differ $(\mathrm{p}=0.114)$. PPT Rate $1,1.62$ (0.223) did not differ from Rate 2, $1.58(0.237) ;(\mathrm{p}=0.356)$ or Rate 3, 1.67 (0.265); ( $\mathrm{p}=0.197)$, but Rate 2 was significantly different from Rate $3(p=0.003)$.

Participant self-reported fatigue (mean rank) did not differ between DRT (5) and PPT (5) $(p=0.755)$. No catheter dislodgements occurred during the trial. 
Table 1 Participant demographic data from postintervention trial questionnaire

\begin{tabular}{lc}
\hline Characteristic & Responses (\%) \\
\hline Participant age range (years) & \\
$<20$ & $0(0)$ \\
$20-29$ & $5(31.3)$ \\
$30-39$ & $6(37.5)$ \\
$40-49$ & $4(25)$ \\
$\geq 50$ & $1(6.3)$ \\
Participant profession & \\
Nurse & $13(81.3)$ \\
Nursing student & $0(0)$ \\
Staff physician & $1(6.3)$ \\
Resident/fellow & $2(15.5)$ \\
Medical student & $0(0)$ \\
Participant student status & \\
Yes & $1(6.3)$ \\
No & $15(93.8)$ \\
Resuscitation experience & \\
None & $1(6.3)$ \\
Minimal & $1(6.3)$ \\
Some experience & $5(31.3)$ \\
Experienced & $6(37.5)$ \\
Very experienced & $3(18.8)$ \\
Use of syringes during a paediatric fluid resuscitation \\
Yes & $15(93.8)$ \\
No & $1(6.3)$ \\
Participant's preferred bolus method in paediatric fluid \\
resuscitation & \\
Regular intravenous pump & $1(6.3)$ \\
Syringe (DRT) & $14(87.5)$ \\
Syringe (PPT) & $1(6.3)$ \\
Rapid infuser & $0(0)$ \\
Pressure bag & $0(0)$ \\
Other & $0(0)$ \\
Do not know & $0(0)$ \\
\hline DRT, disconnect-reconnect Technique; PPT, push-pull \\
Technique. & \\
&
\end{tabular}

Three technical issues were noted during performance of PPT, however none occurred with DRT. One participant performing PPT accidentally drew air into the line, leading to a procedural delay of greater than $60 \mathrm{~s}$. Two additional participants made technical errors while performing PPT: one drew back on the syringe plunger while the stopcock was open to the simulated patient, while a second incorrectly administered fluid to the bag of saline rather than the simulated patient.

\section{DISCUSSION}

This study demonstrates that use of the DRT technique allows for a faster rate of fluid administration than PPT. While fluid resuscitation speed was the primary outcome in this study, other measures of efficiency such as volume of fluid effectively delivered and catheter dislodgement events did not differ between the two techniques under study. Together these findings provide practical information for healthcare providers who must select a method of intravenous fluid administration when faced with a child who requires rapid intravascular volume expansion. While statistically significant, these findings are of unknown clinical importance suggesting a need for further research in this area.

Our previous work demonstrated that healthcare providers experience increasing fatigue with the ongoing performance of manual fluid resuscitation, ${ }^{19}$ and so it was anticipated that a similar finding would be observed in the present study. Contrary to our hypothesis and opposite to our previous findings, fluid administration rate actually increased between the first and second $300 \mathrm{~mL}$ boluses for DRT, and between the second and third boluses for PPT. This unexpected improvement in manual fluid resuscitation performance over the course of the intervention may have been due to a learning effect, despite our use of a standardisation procedure. If a learning effect was indeed present, then it is also interesting that this was observed early on with DRT and later with PPT, suggesting that skill in performing PPT is more difficult to acquire.

The idea that PPT may be challenging for healthcare providers to perform under stressful conditions is further corroborated by our finding of multiple technical issues during performance of the intervention with PPT. In contrast, no technical issues were observed with DRT. We witnessed three technical errors when healthcare providers performed PPT: (1) air drawn into the intravenous line and inability of the provider to problem solve, (2) stopcock toggling error leading to an attempt to 'pull' from the patient instead of the bag and (3) stopcock toggling error leading to an attempt to 'push' fluid back into the bag of saline rather than into the patient. We have in fact observed stopcock toggling errors with performance of PPT in the setting of real resuscitations, which is why we chose to evaluate this outcome in our study. Intravascular air injection can lead to pulmonary air embolism resulting in ventilationperfusion mismatching, right-heart strain and total cardiovascular collapse. ${ }^{22-24}$ In the setting of congenital heart disease systemic air embolism may occur, leading to serious sequelae including stroke. ${ }^{22}$ Stopcock toggling errors are also problematic in that these may lead to delays in fluid administration or jeopardise the integrity of the intravenous catheter if blood is withdrawn. Together these findings would also favour selection of DRT when rapid manual fluid administration is required.

It is important to note that DRT is a two-provider technique. In situations where limited healthcare personnel are available to assist with resuscitation, the use of DRT instead of PPT may interfere with a second provider's availability to perform other simultaneously required vital tasks. Fluid resuscitation is also often performed in the prehospital environment, and use of the DRT technique may be less practical than PPT in a moving ambulance or helicopter. There is also, theoretically, a greater risk of introducing infection with use of DRT, which 


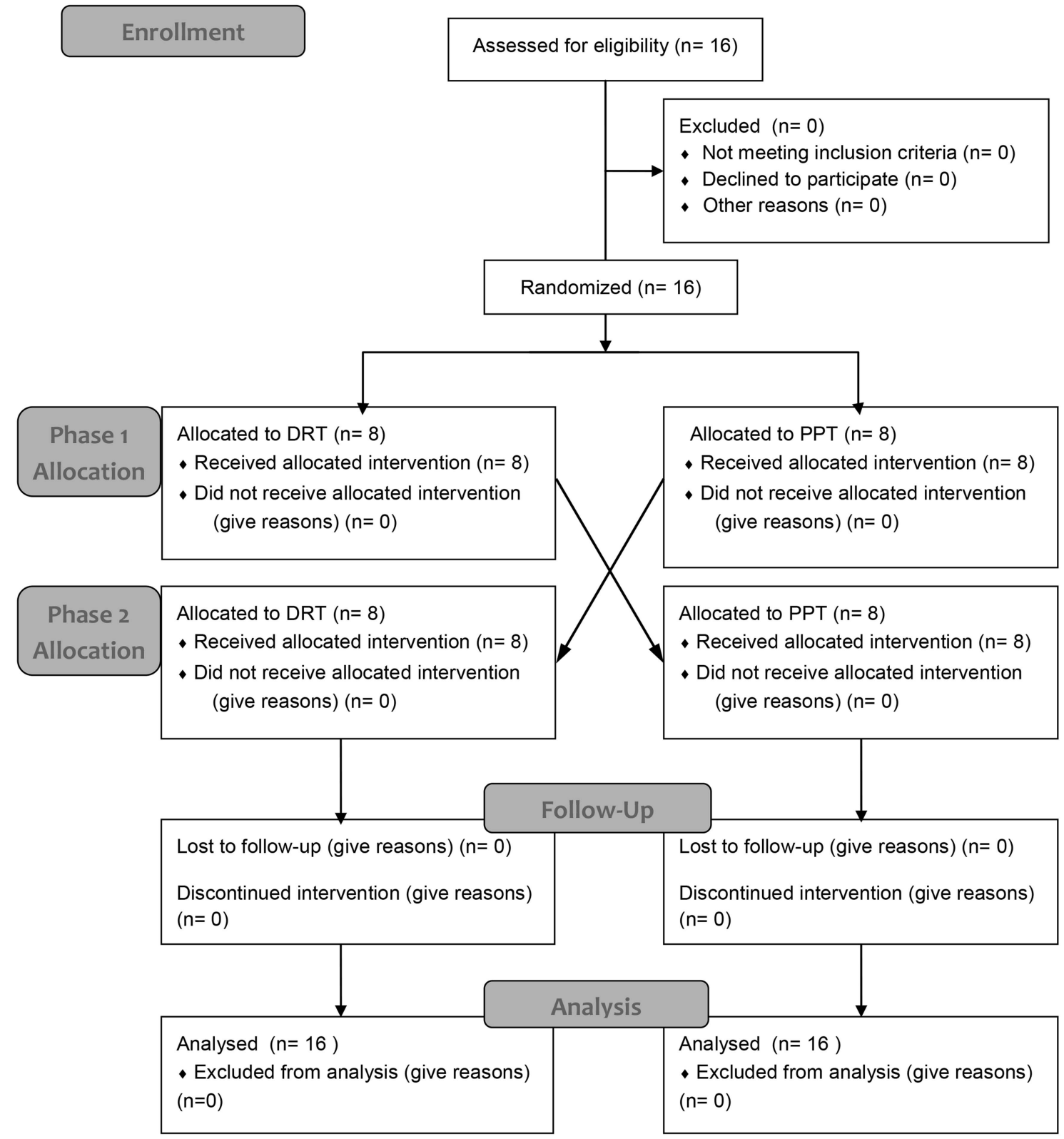

Figure 3 CONSORT trial flow diagram.

requires repeated syringe connection and disconnection from the intravenous line. Studies of central line infection suggest that catheter hubs can be an important infectious source, and that aseptic technique (which may not occur in an urgent scenario) effectively reduces risk. ${ }^{25}$ Since DRT requires the use of multiple syringes, the technique also entails a slightly increased cost and production of waste relative to PPT. Administration of a $300 \mathrm{~mL}$ bolus requires five $60 \mathrm{~mL}$ syringes for DRT versus one syringe for PPT, although DRT does not require use of a triple stopcock. Table 3 provides a summary of the advantages and limitations to consider for the two manual fluid resuscitation techniques evaluated in this study.

The majority of participants in this study were staff nurses. Participant career stage ranged from trainee to experienced staff member, and all but one were familiar with the use of manual syringe techniques for performing fluid resuscitation. Our study population is therefore a good sample of the healthcare providers who would be asked to perform fluid resuscitation in a paediatric tertiary care setting. We would expect healthcare providers working at smaller or non-specialised centres to have less experience with paediatric fluid resuscitation techniques compared with our participants.

There are a number of limitations of our study that warrant mention. All materials were set up in a clinically appropriate fashion for the participants beforehand, unlike in a real resuscitation. While the setup time for each technique was similar, this was not specifically evaluated in our trial. Second, the testing environment was quiet with no interruptions. A lack of distractors may 
Table 2 Outcome analysis results reported with statistical significance

\begin{tabular}{|c|c|c|c|}
\hline Study outcomes & $\begin{array}{l}\text { Disconnect-reconnect } \\
\text { technique (DRT) } \\
n=16 \\
\text { Mean (SD) }\end{array}$ & $\begin{array}{l}\text { Push-pull } \\
\text { technique (PPT) } \\
n=16 \\
\text { Mean (SD) }\end{array}$ & $\begin{array}{l}\text { Effect estimate } \\
\text { Mean difference } \\
(95 \% \mathrm{Cl}) ; \mathrm{p}\end{array}$ \\
\hline \multicolumn{4}{|l|}{ Primary outcome } \\
\hline Overall fluid infusion rate (mL/s) & $1.77(0.145)$ & $1.62(0.226)$ & 0.153 (0.055 to 0.251$) ; 0.005$ \\
\hline \multicolumn{4}{|l|}{ Secondary outcomes } \\
\hline \multirow[t]{3}{*}{ Fluid infusion rate by bolus $(\mathrm{mL} / \mathrm{s})^{*}$} & Bolus 1:1.63 (0.143) & Bolus 1:1.62 (0.223) & $0.016(-0.088$ to 0.121$) ; 0.744$ \\
\hline & Bolus 2:1.83 (0.176) & Bolus 2:1.58 (0.356) & $0.246(0.136$ to 0.357$) ;<0.001$ \\
\hline & Bolus $3: 1.88(0.180)$ & Bolus 3:1.67 (0.265) & 0.214 (0.087 to 0.340$) ; 0.003$ \\
\hline Total fluid volume infused (mL) & $891.8(36.60)$ & $898.13(58.11)$ & $-6.250(-52.760$ to 40.260$) ; 0.778$ \\
\hline Subjective fatigue rank (mean rank) & $5.75(1.0)$ & $5.63(1.20)$ & $0.125(-0.650$ to 0.900$) ; 0.736$ \\
\hline Median (Q1, Q3) & $6.0(5.0,6.5)$ & $5.5(5.0,7.0)$ & $\mathrm{p}=0.836$ \\
\hline Catheter dislodgement events (n) & 0 & 0 & NA \\
\hline Technical issues encountered (n) & 0 & 3 & NA \\
\hline \multicolumn{4}{|c|}{$\begin{array}{l}{ }^{*} \text { DRT infusion rates differ between bolus } 1,2 \text { and } 3 ; p<0.001 \text {. } \\
\text { DRT infusion rates differ between bolus } 1 \text { and } 2 ; p<0.001 \text {. } \\
\text { DRT infusion rates differ between bolus } 1 \text { and } 3 ; p<0.001 \text {. } \\
\text { DRT infusion rates do not differ between bolus } 2 \text { and bolus } 3 ; p=0.114 \text {. } \\
\text { PPT infusion rates differ between bolus } 1,2 \text { and } 3 ; p=0.003 \text {. } \\
\text { PPT infusion rates differ between bolus } 2 \text { and } 3 ; p=0.003 \text {. } \\
\text { PPT infusion rates did not differ between bolus } 1 \text { and either bolus } 2 ; p=0.356 \text { or bolus } 3 ; p=0.197 \text {. } \\
\text { NA, not applicable. }\end{array}$} \\
\hline
\end{tabular}

have allowed participants to focus more effectively than is possible in practice, leading to greater accuracy and fewer errors. No catheter dislodgments occurred during testing, raising the possibility that our model may have offered some protection from this. However, catheter dislodgement from the model was indeed possible and occurred during pilot testing. Evaluation of PPT in a previous clinical trial found catheter dislodgement events to be a rare occurrence, experienced by only 1/ 57 children. $^{20}$ Finally, although our study demonstrated a statistically significant difference between DRT and PPT fluid administration rates, with the $95 \%$ CI $(0.055 \mathrm{~mL} / \mathrm{s}$ to $0.251 \mathrm{~mL} / \mathrm{s})$ including the $0.2 \mathrm{~mL} / \mathrm{s}$ mean difference this study was powered to detect, our findings are of unknown clinical significance. Notwithstanding this, our work provides new data for the resuscitation community to consider, in light of current paediatric resuscitation guidelines. ${ }^{3-5} 712$

\section{CONCLUSION}

This study demonstrates that DRT facilitates a faster rate of fluid administration than PPT, and that PPT is associated with more technical errors. It may therefore be appropriate to recommend DRT as the preferred method of manual paediatric fluid resuscitation using syringes, although factors such as the patient, environment, resources and availability of personnel will also

Table 3 Advantages and limitations of two provider-endorsed manual paediatric fluid resuscitation techniques

\begin{tabular}{|c|c|c|}
\hline Technique & Advantages & Limitations \\
\hline $\begin{array}{l}\text { Disconnect- } \\
\text { reconnect (DRT) }\end{array}$ & $\begin{array}{l}\text { Facilitates a faster rate of fluid administration } \\
\text { Simple technique; likely easier to learn and } \\
\text { possibly easier to recall and perform }\end{array}$ & $\begin{array}{l}\text { Requires two providers } \\
\text { Many syringes required which may increase } \\
\text { cost and waste production } \\
\text { Requires multiple connections to the } \\
\text { intravenous line which may increase risk of } \\
\text { contamination }\end{array}$ \\
\hline Push-pull (PPT) & $\begin{array}{l}\text { Can be performed by a single provider } \\
\text { Likely better suited to space-limited } \\
\text { environments, such as the out-of-hospital setting } \\
\text { May require only a single connection to the } \\
\text { intravenous line (closed system) which may } \\
\text { decrease risk of contamination }\end{array}$ & $\begin{array}{l}\text { Facilitates a slower rate of fluid administration } \\
\text { More complex task; requires greater dexterity } \\
\text { and more practice may be needed for optimal } \\
\text { recall and performance } \\
\text { Risk of adverse events as a result of stopcock } \\
\text { toggling errors }\end{array}$ \\
\hline
\end{tabular}


affect provider choices. Further study in the clinical setting is required to support recommendations in future iterations of paediatric resuscitation guidelines regarding the safest and most effective way to perform rapid fluid resuscitation for children.

\author{
Author affiliations \\ ${ }^{1}$ Department of Pediatrics, McMaster Children's Hospital and McMaster \\ University, Hamilton, Ontario, Canada \\ ${ }^{2}$ Department of Clinical Epidemiology and Biostatistics, McMaster University, \\ Hamilton, Ontario, Canada \\ ${ }^{3}$ Biostatistics Unit,/FSORC, St Joseph's Healthcare Hamilton, Hamilton, \\ Canada \\ ${ }^{4}$ Department of Anesthesia, McMaster University, Hamilton, Ontario, Canada \\ ${ }^{5}$ Division of Emergency Medicine, Department of Pediatrics, The Hospital for \\ Sick Children, and University of Toronto, University Avenue, Toronto, Ontario, \\ Canada
}

Acknowledgements The authors thank participating healthcare providers from McMaster Children's Hospital for their time and effort, without which this trial could not have been completed. Mark Duffett organised the randomisation schedule and assisted with randomisation of trial participants. Michael Chong and Zach Arnott volunteered their time and served as our blinded video reviewers. Dr Lawrence Mbuagbaw (PhD) provided assistance with statistical analyses.

Contributors ETC developed the trial protocol including study objectives, under the mentorship of MJP and with input from GH. ETC also prepared the REB submission and produced revisions as required. ETC and GH trained the research assistants and assisted with participant recruitment. ETC was responsible for input of results data to the study database. ETC was involved with data analysis and interpretation of study results, and produced the first version of this manuscript. GH assisted in revision of the manuscript draft, and participated in all stages of trial planning, document production and analysis. SU was primarily responsible for participant recruitment and scheduling. She ensured all testing was conducted appropriately, with assistance from ETC and MJP. GF and LT participated in trial design and statistical analysis planning. GF performed the sample size calculations. LT was responsible for the analysis of the data. MJP conceived of the research question, played a major role in development of study objectives, and was responsible for scientific oversight of the trial. She played a key role in protocol development, preliminary data analysis and interpretation. She also revised the first draft of the manuscript and played a supervisory role in overseeing the work of ECT, GH and SU. All authors reviewed and contributed to the submitted version of the paper.

Funding This work was supported by the following funding sources. A CIHR Health Professional Student Research Award supported ETC for his work on this study. A Regional Medical Associates Research Scholarship (2013H00101) supported GH by providing operating funds for trial conduct. MJP is supported by a Hamilton Health Sciences Research Early Career Award (no assigned grant number). New Faculty Start-up Funding from McMaster University (held by MP) also supported conduct of this work. None of the funding sources were in any way involved with trial design, conduction or data analysis.

\section{Competing interests None.}

Ethics approval Hamilton Integrated Research Ethics Board (formerly Hamilton Health Sciences Research Ethics Board).

Provenance and peer review Not commissioned; externally peer reviewed.

Data sharing statement The data presented in this manuscript represent the analysis of all the available data related to this work. The primary data set for this study is available to the corresponding author, MP. Study participants did not consent to the publication of the full data set.

Open Access This is an Open Access article distributed in accordance with the Creative Commons Attribution Non Commercial (CC BY-NC 3.0) license, which permits others to distribute, remix, adapt, build upon this work non- commercially, and license their derivative works on different terms, provided the original work is properly cited and the use is non-commercial. See: http:// creativecommons.org/licenses/by-nc/3.0/

\section{REFERENCES}

1. Yager P, Noviski N. Shock. Pediatr Rev 2010;31:311-18.

2. McKiernan CA, Lieberman SA. Circulatory shock in children: an overview. Pediatri Rev 2005;26:451-60.

3. Management of shock. In: Chameides L, Samson RA, Schexnayder SMHazinski MF, eds. Pediatric advanced life support provider manual. Dallas, TX: American Heart Association, 2011:85-108.

4. Shock. Advanced trauma life support for doctors student manual. 8th edn. Chicago, IL: American College of Surgeons Committee on Trauma, 2008:55-71.

5. Pediatric Trauma. Advanced trauma life support for doctors student course manual. 8th edn. Chicago, IL: American College of Surgeons Committee on Trauma, 2008:225-46.

6. Nolan JP, Soar J, Zideman DA, et al. European resuscitation council guidelines for resuscitation 2010. Resuscitation 2010;81:1219-76.

7. Brierley J, Carcillo JA, Choong K, et al. Clinical practice parameters for hemodynamic support of pediatric and neonatal septic shock: 2007 update from the American College of Critical Care Medicine. Crit Care Med 2009;37:666-88.

8. Maitland K, Kiguli S, Opoka RO, et al. Mortality after fluid bolus in African children with severe infection. N Engl J Med 2011;364:2483-95.

9. Ford N, Hargreaves S, Shanks L. Mortality after fluid bolus in children with shock due to sepsis or severe infection: a systematic review and meta-analysis. PLOS ONE 2012;7:e43953.

10. Dellinger RP, Levy MM, Rhodes A, et al. Surviving sepsis campaign international guidelines for the management of severe sepsis and septic shock. Crit Care Med 2013;41:580-637.

11. Carcillo JA, Davis AL, Zaritsky A. Role of early fluid resuscitation in pediatric septic shock. JAMA 1991;266:1242-5.

12. Han YY, Carcillo JA, Dragotta MA, et al. Early reversal of pediatric-neonatal septic shock by community physicians is associated with improved outcome. Pediatrics 2003;112:793-9.

13. Oliveira CF, Nogueira de Sa FR, Oliveira DSF, et al. Time- and fluid-sensitive resuscitation for hemodynamic support of children in septic shock: barriers to the implementation of the American College of Critical Care Medicine/Pediatric Advanced Life Support Guidelines in a pediatric intensive care unit in a developing world. Pediatr Emerg Care 2008;24:810-15.

14. Ranjit S, Kissoon N, Jayakumar I. Aggressive management of dengue shock syndrome may decrease mortality rate: a suggested protocol. Pediatr Crit Care Med 2005;6:412-19.

15. Carcillo JA, Tasker RC. Fluid resuscitation of hypovolemic shock: acute medicine's great triumph for children. Intens Care Med 2006;32:958-61.

16. Inwald DP, Tasker RC, Peters MJ, et al. Emergency management of children with severe sepsis in the United Kingdom: the results of the Paediatric Intensive Care Society sepsis audit. Arch Dis Child 2009;94:348-53.

17. Paul R, Neuman MI, Monuteaux MC, et al. Adherence to PALS sepsis guidelines and hospital length of stay. Pediatrics 2012;130:e273.

18. Parker MJ, Manan A. Translating resuscitation guidelines into practice: health care provider attitudes, preferences and beliefs regarding pediatric fluid resuscitation performance. PLOS ONE 2013;8:e58282.

19. Harvey G, Foster G, Manan A, et al. Factors affecting pediatric isotonic fluid resuscitation efficiency: a randomized controlled trial evaluating the impact of syringe size. BMC Emerg Med 2013;13:14.

20. Stoner MJ, Goodman DG, Cohen DM, et al. Rapid fluid resuscitation in pediatrics: testing the American College of Critical Care Medicine guideline. Ann Emerg Med 2007;50:601-7.

21. Cole ET, Harvey G, Foster G, et al. Study protocol for a randomised controlled trial comparing the efficiency of two provider-endorsed manual paediatric fluid resuscitation techniques. BMJ Open 2013;21:e002754.

22. Orebaugh S. Venous air embolism: clinical and experimental considerations. Crit Care Med 1992;20:1169-77.

23. Athar MK, Islam T, Grammes J, et al. Near-fatal pulmonary air embolus from iatrogenic injection of air during contrast administration. QJM 2005;98:231-2.

24. Palmon SC, Moore LE, Lundberg J, et al. Venous air embolism: a review. J Clin Anesth 1997;9:251-7.

25. Salzman MB, Isenberg HD, Rubin LG. Use of disinfectants to reduce microbial contamination of hubs of vascular catheters. $J$ Clin Microbiol 1993;31:475-9. 